\title{
Brain structural abnormalities in obesity: relation to age, genetic risk, and common psychiatric disorders
}

\author{
Evidence through univariate and multivariate mega-analysis including 6420 participants from \\ the ENIGMA MDD working group
}

Nils Opel ${ }^{1,2} \cdot$ Anbupalam Thalamuthu $^{3,4} \cdot$ Yuri Milaneschi ${ }^{5} \cdot$ Dominik Grotegerd $^{1} \cdot$ Claas Flint $^{1,6}$. Ramona Leenings $^{1} \cdot$ Janik Goltermann ${ }^{1} \cdot$ Maike Richter $^{1} \cdot$ Tim Hahn $^{1} \cdot$ Georg Woditsch $^{7} \cdot$ Klaus Berger $^{8} \cdot$ Marco Hermesdorf $^{8} \cdot$ Andrew Mclntosh $\left.^{9}\right)^{9}$ Heather C. Whalley $^{9} \cdot$ Mathew A. Harris $^{9} \cdot$ Frank P. MacMaster $^{10,11}$. Henrik Walter $\mathbb{D}^{12} \cdot$ Ilya M. Veer ${ }^{12}$. Thomas Frodl ${ }^{13,14} \cdot$ Angela Carballedo $^{13} \cdot$ Axel Krug $^{15} \cdot$ Igor Nenadic $^{15} \cdot$ Tilo Kircher ${ }^{15}$. Andre Aleman ${ }^{16} \cdot$ Nynke A. Groenewold ${ }^{16} \cdot{\text { Dan J. Stein }{ }^{17} \text { - Jair C. Soares }}^{18}$. Giovana B. Zunta-Soares ${ }^{18} \cdot$ Benson Mwangi $^{19} \cdot$ Mon-Ju Wu $\mathbb{D}^{19} \cdot$ Martin Walter $^{20} \cdot$ Meng Li $^{20} \cdot$ Ben J. Harrison $^{21}$. Christopher G. Davey (D) $^{22,23} \cdot$ Kathryn R. Cullen ${ }^{24} \cdot$ Bonnie Klimes-Dougan $^{25} \cdot$ Bryon A. Mueller (iD $^{24}$. Philipp G. Sämann ${ }^{26}$ - Brenda Penninx ${ }^{5}$ Laura Nawijn (D) $^{5} \cdot$ Dick J. Veltman $^{5}$ Lyubomir Aftanas ${ }^{27} \cdot$ Ivan V. Brak $^{27}$. Elena A. Filimonova ${ }^{27} \cdot$ Evgeniy A. Osipov $\mathbb{D}^{28} \cdot$ Liesbeth Reneman $^{29} \cdot$ Anouk Schrantee $^{29} \cdot$ Hans $^{3}$. Grabe ${ }^{30,31}$. Sandra Van der Auwera ${ }^{30,31} \cdot$ Katharina Wittfeld $\mathbb{D}^{30,31} \cdot$ Norbert Hosten $^{32} \cdot$ Henry Völzke H,34 $^{33, K^{3}}$ Sang Sim ${ }^{35,36}$. Ian H. Gotlib ${ }^{37}$ - Matthew D. Sacchet ${ }^{38}$ - Jim Lagopoulos ${ }^{39} \cdot$ Sean N. Hatton (D) $^{40}$ Ian Hickie $^{40}$ - Elena Pozzi $^{22,41}$. Paul M. Thompson ${ }^{42} \cdot$ Neda Jahanshad (iD ${ }^{42} \cdot$ Lianne Schmaal $^{22,23} \cdot$ Bernhard T. Baune $^{1,43,44} \cdot$ Udo Dannlowski $^{1}$

Received: 20 October 2019 / Revised: 28 April 2020 / Accepted: 30 April 2020 / Published online: 28 May 2020

(c) The Author(s) 2020. This article is published with open access, corrected publication 2021

\begin{abstract}
Emerging evidence suggests that obesity impacts brain physiology at multiple levels. Here we aimed to clarify the relationship between obesity and brain structure using structural MRI $(n=6420)$ and genetic data ( $n=3907)$ from the ENIGMA Major Depressive Disorder (MDD) working group. Obesity (BMI > 30) was significantly associated with cortical and subcortical abnormalities in both mass-univariate and multivariate pattern recognition analyses independent of MDD diagnosis. The most pronounced effects were found for associations between obesity and lower temporo-frontal cortical thickness (maximum Cohen's $d$ (left fusiform gyrus) $=-0.33$ ). The observed regional distribution and effect size of cortical thickness reductions in obesity revealed considerable similarities with corresponding patterns of lower cortical thickness in previously published studies of neuropsychiatric disorders. A higher polygenic risk score for obesity significantly correlated with lower occipital surface area. In addition, a significant age-by-obesity interaction on cortical thickness emerged driven by lower thickness in older participants. Our findings suggest a neurobiological interaction between obesity and brain structure under physiological and pathological brain conditions.
\end{abstract}

These authors contributed equally: Bernhard T. Baune, Udo Dannlowski

Supplementary information The online version of this article (https:// doi.org/10.1038/s41380-020-0774-9) contains supplementary material, which is available to authorized users.

Nils Opel

n_ope101@uni-muenster.de

Extended author information available on the last page of the article

\section{Introduction}

With an estimated worldwide prevalence of $13 \%$ among the adult population and up to $38 \%$ in western societies [1], obesity is one of the greatest concerns to public health [2]. The role of obesity as a preventable cardiovascular risk factor is well known, but research has only recently started to explore the neurobiological underpinnings of obesity.

On a systemic level, neuroimaging research has identified structural [3-5] and functional [6-8] alterations in obese participants - one of the most consistent findings is 
decreased gray matter volume in obesity $[3,4,9,10]$. A recent UK Biobank study including data from $n=9652$ participants supplemented this notion by showing an inverse association between BMI and global gray matter volume [11]. Further large-scale evidence for associations between body weight and brain structure comes from a recent meta-analysis of voxel-based morphometry studies including data from $n=5882$ subjects that pointed to consistent associations between BMI and lower gray matter volume in the medial prefrontal cortex, the bilateral cerebellum, and the left temporal pole [12]. However, even though these well-powered studies provide robust evidence for an association between BMI and brain structure in general, the current understanding of the relationship between obesity and brain structure is considerably limited for several reasons.

First, the distribution and effect size of brain structural abnormalities in obesity remains unclear. Several smaller structural neuroimaging studies suggest that obesity might primarily relate to gray matter reductions in brain areas involved in reward processing and impulse regulation such as the orbitofrontal cortex and the striatum [9, 13, 14]. Even so, other reports question the hypothesis of regional specific gray matter decrease in obesity by pointing to widespread associations throughout the brain with diverging effects of obesity on subcortical brain structure $[4,10]$. Since prior studies either exhibited limited power to detect subtle effects in small samples or employed hypothesis-driven region of interest approaches, the distribution or regional specificity of obesity-related brain structural abnormalities remains uncertain. Large-scale studies are needed that investigate associations with obesity throughout the entire brain by differentiating effects on subcortical volume and cortical thickness and surface area. Furthermore, while the statistical significance of obesity-related brain structural abnormalities is well documented, the effect sizes and hence the potential relevance of brain structural alterations in obesity remains unknown. We aimed to address this issue by directly comparing profiles of obesity-related brain structural alterations with findings from neuropsychiatric disorders. In addition we aimed to complement group level analyses, by employing individual-level based pattern classification as a further proxy for the robustness of neuroimaging findings [15]. Second, previous neuroimaging findings in obesity are largely based on studies in healthy participants. Yet, obesity has frequently been associated with neuropsychiatric disorders $[16,17]$ and more specifically previous research has pointed to a bidirectional association between obesity and major depression [18]. Furthermore, preliminary neuroimaging studies have reported overlapping brain structural abnormalities in obesity and major depression [9, 12, 19]. It thus appears relevant to investigate if obesity-related brain structural abnormalities might similarly be present under physiological and pathological brain conditions. Against this backdrop, the present study aimed to provide a wellpowered and comprehensive investigation of the relationship between obesity and brain structural abnormalities in healthy participants and depressive patients. A third major issue concerns the relationship between brain structural abnormalities in obesity and ageing. Interestingly, while obesity and gray matter volume are frequently reported to be inversely related in adult samples, the few studies of obesity-related brain structural abnormalities in children and adolescents have diverging results $[13,20,21]$. Thus, it is valuable to investigate whether brain structural impairment in obesity is already detectable in children and adolescents and if brain structural abnormalities in obesity might vary as a function of age. In addition, there may be a genetic contribution to brain structural abnormalities in obesity, given the high heritability of obesity in general [22] and the involvement of multiple BMI-related genetic variants in brain physiology [23]. Thus, the question of a potential genetic contribution to brain structural abnormalities in obesity arises. To address this, we combined individual polygenic risk profiles with imaging data to investigate obesity and BMI-related brain structural abnormalities [24, 25].

\section{Methods}

\section{Participants}

We studied BMI and neuroimaging data in a combined sample of 6420 participants (mean age $=42.91, \mathrm{SD}=15.26$; $56.95 \%$ female; mean $\mathrm{BMI}=25.97, \mathrm{SD}=4.97$ ) including healthy controls (HC: $n=3519$ ) and major depressive disorder patients (MDD: $n=2901$ ) from 28 sites contributing to the ENIGMA MDD working group $[19,26]$. The sample included $n=1223$ obese participants (BMI $>30$ ) as well as $n=2917$ normal weight participants (BMI 18.5-25) (Supplementary Results, Supplementary Figs. 1, 2, 3, Supplementary Tables 1,2). All participating sites obtained approval from local institutional review boards and ethics committees; all study participants provided written informed consent.

\section{Structural MRI methods}

T1-weighted high-resolution anatomical brain images were acquired for all participants and preprocessed locally using FreeSurfer segmentation. Quality control was carried out at each site according to protocols from the ENIGMA consortium. Segmentation quality was assessed by visual inspection and statistically evaluated for outliers with a standardized protocol provided by the ENIGMA consortium (http://enigma.ini.usc.edu/protocols/imagingprotocols). Details of the imaging procedures for each 
cohort may be found in the Supplementary material (Supplementary Table 3). All structural images were preprocessed using the subcortical and cortical parcellation stream of FreeSurfer with the default parameters [27]. As we aimed to provide a comprehensive overview of obesityrelated brain structural alterations that would allow for comparison with previous ENIGMA studies, all available imaging measures were included for the presented analyses: global measures included total intracranial volume, total left and right cortical surface area, and average left and right cortical thickness. Regional measures included subcortical volumetric measures ( 8 left and 8 right), surface area (34 left and 34 right), and thickness measures (34 left and 34 right) for all cortical regions based on the Desikan-Killiany atlas [28]. The presented morphometric data allowed us to simultaneously investigate both subcortical and cortical abnormalities and furthermore enabled us to examine thickness and surface area separately which have been shown to be driven by distinct genetic mechanisms and to exhibit different developmental trajectories [29, 30].

\section{Genetic methods}

Genetic data were available for 3907 individuals from nine contributing sites. Genotyping of these subjects was performed at each contributing site using published protocols (Supplementary Table 4). Polygenic risk scores (PRS) were generated using sets of SNPs selected based on $p$ value thresholds at $p=[0.1 ; 0.2 ; 0.3 ; 0.4 ; 0.5 ; 0.6 ; 0.7 ; 0.8 ; 0.9$; 1.0] from the base GWAS data. The R program 'PRSice' [31]—which uses PLINK-1.9 [32] in the background for linkage disequilibrium pruning - was used for this analysis step. Standardized PRS values based on z-transformation were used for all analyses (Supplementary Methods).

\section{Statistical analyses}

All univariate imaging analyses were carried out using linear models in R, separately for each of the 157 available FreeSurfer derived imaging measures as a dependent variable. Age, sex, MDD diagnosis, and site were included as covariates in all models. For analyses of subcortical volumes and surface area measures, ICV was also included as covariate. For all univariate imaging analyses, FDR correction for 157 tests was conducted using the Benjamini Hochberg procedure with a false discovery rate of $q<0.05$.

To investigate associations between brain structure and obesity, two main models were applied by including a dichotomous predictor based on a BMI threshold (obese subjects $(\mathrm{BMI}>30)$ versus normal weight subjects (BMI 18.5-25) (Model A)) and furthermore by including BMI as a continuous predictor (Model B).
Effect size estimates (Cohen's $d$ ) were calculated based on $t$-values and sample sizes [33] from the regression model including the dichotomous BMI group (obesity versus normal weight) predictor (Model A) thus following a similar methodology compared with previous studies on psychiatric disorders from the ENIGMA consortium [19, 26]. To investigate potential similarities between brain structural alterations in obesity and common neuropsychiatric disorders, we carried out correlational analyses between effect size estimates (Cohen's $d$ ) of thickness alterations in all cortical regions in obesity with effect size estimates reported in previous ENIGMA studies on MDD [19] and bipolar disorder [34].

To further test our hypothesis of brain structural alterations in obesity, we complemented the applied massunivariate testing approach by conducting pattern recognition analyses to investigate multivariate patterns of brain structural differences between obese and normal weight subjects. To this end, a machine learning pipeline consisting of several preprocessing steps including imputation of missing values, dimensionality reduction by principal component analysis and random undersampling and a support vector machine was trained on all available 157 FreeSurfer derived imaging measures to individually classify participants as either obese or normal weight using pooled multisite nested cross-validation employing the PHOTON framework (https://photon-ai.com; Supplementary Methods).

Furthermore, potential interaction effects of body weight and age, sex and MDD diagnosis were carried out as exploratory analyses. In addition, associations between polygenic risk for obesity and brain structure were assessed through univariate models as outlined above.

\section{Results}

\section{Obesity and brain structure}

Linear regression models including either obesity as dichotomous predictor (Model A) or BMI as continuous predictor (Model B) of brain structure yielded highly consistent results (Supplementary Tables 5, 6, Supplementary Fig. 4). Obesity was associated with lower cortical thickness, with most pronounced and consistent associations between obesity and lower cortical thickness in regions of the temporal and frontal lobe (Table 1 and Fig. 1). Analyses of regionally specific cortical surface area alterations in obesity revealed both significantly lower and higher surface area in obese subjects. Subcortical volumes were found to be significantly increased in obese subjects-with most pronounced volume increases in the amygdala, the thalamus and the nucleus accumbens (Table 1). 
Table 1 FDR-corrected significant results for group differences between obese and normal weight subjects as assessed using separate linear regression models with a dichotomous group predictor (obesity versus normal weight).

\begin{tabular}{|c|c|c|c|c|c|c|c|c|}
\hline Label & Estimate & Std error & $T$ & $p$ & FDR adjusted $p$ & Cohen's $d$ & $N$ Obese & $N \mathrm{NW}$ \\
\hline \multicolumn{9}{|l|}{ Global measures } \\
\hline Left hemispheral average thickness & -0.021 & 0.003 & -6.23 & $5.18 \mathrm{E}-10$ & $<0.0001$ & -0.214 & 1200 & 2865 \\
\hline Right hemispheral average thickness & -0.020 & 0.003 & -5.89 & $4.18 \mathrm{E}-09$ & $<0.0001$ & -0.203 & 1200 & 2865 \\
\hline Total Intracranial Volume & -21634.000 & 5603.000 & -3.86 & $1.10 \mathrm{E}-04$ & 0.0005 & -0.135 & 1168 & 2755 \\
\hline Total right hemispheral surface area & -708.380 & 258.090 & -2.74 & $6.08 \mathrm{E}-03$ & 0.0165 & -0.095 & 1189 & 2872 \\
\hline Total left hemispheral surface area & -654.300 & 256.890 & -2.55 & $1.09 \mathrm{E}-02$ & 0.0281 & -0.088 & 1189 & 2872 \\
\hline \multicolumn{9}{|l|}{ Cortical thickness } \\
\hline Left fusiform gyrus & -0.051 & 0.005 & -9.59 & $2.00 \mathrm{E}-16$ & $<0.0001$ & -0.331 & 1195 & 2849 \\
\hline Right fusiform gyrus & -0.050 & 0.005 & -9.42 & $2.00 \mathrm{E}-16$ & $<0.0001$ & -0.325 & 1193 & 2849 \\
\hline Right superior temporal gyrus & -0.041 & 0.006 & -7.17 & $9.09 \mathrm{E}-13$ & $<0.0001$ & -0.251 & 1161 & 2745 \\
\hline Left superior temporal gyrus & -0.040 & 0.006 & -6.88 & $7.04 \mathrm{E}-12$ & $<0.0001$ & -0.243 & 1138 & 2684 \\
\hline Left inferior temporal gyrus & -0.040 & 0.006 & -6.62 & $4.17 \mathrm{E}-11$ & $<0.0001$ & -0.231 & 1165 & 2823 \\
\hline Left middle temporal gyrus & -0.039 & 0.006 & -6.46 & $1.18 \mathrm{E}-10$ & $<0.0001$ & -0.227 & 1149 & 2748 \\
\hline Right middle temporal gyrus & -0.036 & 0.006 & -6.06 & $1.49 \mathrm{E}-09$ & $<0.0001$ & -0.210 & 1184 & 2815 \\
\hline Right pars opercularis & -0.033 & 0.006 & -5.96 & $2.70 \mathrm{E}-09$ & $<0.0001$ & -0.206 & 1189 & 2835 \\
\hline Right posterior cingulate cortex & -0.033 & 0.006 & -5.96 & $2.71 \mathrm{E}-09$ & $<0.0001$ & -0.205 & 1196 & 2859 \\
\hline Right inferior temporal gyrus & -0.036 & 0.006 & -5.88 & $4.54 \mathrm{E}-09$ & $<0.0001$ & -0.204 & 1175 & 2838 \\
\hline Left precentral gyrus & -0.030 & 0.005 & -5.85 & $5.27 \mathrm{E}-09$ & $<0.0001$ & -0.202 & 1192 & 2837 \\
\hline Right precentral gyrus & -0.030 & 0.005 & -5.76 & $9.13 \mathrm{E}-09$ & $<0.0001$ & -0.199 & 1188 & 2844 \\
\hline Right superior frontal gyrus & -0.030 & 0.005 & -5.76 & $8.93 \mathrm{E}-09$ & $<0.0001$ & -0.199 & 1189 & 2859 \\
\hline Left transverse temporal gyrus & -0.042 & 0.008 & -5.29 & $1.26 \mathrm{E}-07$ & $<0.0001$ & -0.182 & 1195 & 2853 \\
\hline Left insula & -0.030 & 0.006 & -5.17 & $2.41 \mathrm{E}-07$ & $<0.0001$ & -0.179 & 1188 & 2811 \\
\hline Left posterior cingulate cortex & -0.030 & 0.006 & -5.16 & $2.56 \mathrm{E}-07$ & $<0.0001$ & -0.178 & 1196 & 2857 \\
\hline Right medial orbitofrontal cortex & -0.031 & 0.006 & -5.12 & $3.18 \mathrm{E}-07$ & $<0.0001$ & -0.177 & 1183 & 2831 \\
\hline Left banks of the superior temporal sulcus & -0.031 & 0.006 & -4.88 & $1.08 \mathrm{E}-06$ & $<0.0001$ & -0.172 & 1139 & 2708 \\
\hline Left caudal middle frontal gyrus & -0.026 & 0.005 & -4.89 & $1.04 \mathrm{E}-06$ & $<0.0001$ & -0.169 & 1196 & 2840 \\
\hline Right banks of the superior temporal sulcus & -0.030 & 0.006 & -4.63 & $3.81 \mathrm{E}-06$ & $<0.0001$ & -0.161 & 1178 & 2796 \\
\hline Left entorhinal cortex & -0.061 & 0.013 & -4.5 & $6.86 \mathrm{E}-06$ & $<0.0001$ & -0.158 & 1164 & 2725 \\
\hline Left paracentral lobule & -0.024 & 0.005 & -4.46 & $8.55 \mathrm{E}-06$ & $<0.0001$ & -0.154 & 1195 & 2857 \\
\hline Right parahippocampal gyrus & -0.044 & 0.010 & -4.46 & $8.50 \mathrm{E}-06$ & $<0.0001$ & -0.154 & 1192 & 2850 \\
\hline Left temporal pole & -0.059 & 0.014 & -4.38 & $1.20 \mathrm{E}-05$ & 0.0001 & -0.151 & 1187 & 2851 \\
\hline Left superior frontal gyrus & -0.023 & 0.005 & -4.35 & $1.36 \mathrm{E}-05$ & 0.0001 & -0.150 & 1194 & 2851 \\
\hline Left supramarginal gyrus & -0.021 & 0.005 & -4.15 & $3.45 \mathrm{E}-05$ & 0.0002 & -0.145 & 1173 & 2767 \\
\hline Right precuneus & -0.019 & 0.005 & -4.13 & $3.75 \mathrm{E}-05$ & 0.0002 & -0.142 & 1195 & 2848 \\
\hline Left pars opercularis & -0.021 & 0.005 & -4.03 & $5.58 \mathrm{E}-05$ & 0.0003 & -0.139 & 1194 & 2845 \\
\hline Right paracentral lobule & -0.022 & 0.005 & -3.94 & $8.38 \mathrm{E}-05$ & 0.0004 & -0.136 & 1196 & 2857 \\
\hline Right caudal middle frontal gyrus & -0.020 & 0.005 & -3.73 & $2.00 \mathrm{E}-04$ & 0.0008 & -0.129 & 1194 & 2845 \\
\hline Left isthmus cingulate cortex & -0.026 & 0.007 & -3.7 & $2.20 \mathrm{E}-04$ & 0.0009 & -0.128 & 1195 & 2852 \\
\hline Right lateral orbitofrontal cortex & -0.022 & 0.006 & -3.68 & $2.40 \mathrm{E}-04$ & 0.0009 & -0.127 & 1195 & 2858 \\
\hline Left precuneus & -0.017 & 0.005 & -3.66 & $2.60 \mathrm{E}-04$ & 0.0010 & -0.126 & 1189 & 2851 \\
\hline Right temporal pole & -0.050 & 0.014 & -3.58 & $3.40 \mathrm{E}-04$ & 0.0012 & -0.124 & 1191 & 2850 \\
\hline Left lateral orbitofrontal cortex & -0.021 & 0.006 & -3.56 & $3.70 \mathrm{E}-04$ & 0.0013 & -0.123 & 1188 & 2851 \\
\hline Right rostral middle frontal gyrus & -0.017 & 0.005 & -3.53 & $4.10 \mathrm{E}-04$ & 0.0014 & -0.122 & 1192 & 2849 \\
\hline Left inferior parietal cortex & -0.017 & 0.005 & -3.5 & $4.80 \mathrm{E}-04$ & 0.0016 & -0.121 & 1180 & 2831 \\
\hline Right insula & -0.022 & 0.006 & -3.46 & $5.40 \mathrm{E}-04$ & 0.0018 & -0.120 & 1182 & 2777 \\
\hline Right pars triangularis & -0.020 & 0.006 & -3.39 & $7.20 \mathrm{E}-04$ & 0.0023 & -0.117 & 1187 & 2838 \\
\hline
\end{tabular}


Table 1 (continued)

\begin{tabular}{|c|c|c|c|c|c|c|c|c|}
\hline Label & Estimate & Std error & $T$ & $p$ & FDR adjusted $p$ & Cohen's $d$ & $N$ Obese & $N \mathrm{NW}$ \\
\hline Right isthmus cingulate cortex & -0.022 & 0.007 & -3.18 & $1.50 \mathrm{E}-03$ & 0.0045 & -0.110 & 1196 & 2854 \\
\hline Right supramarginal gyrus & -0.016 & 0.005 & -3.18 & $1.50 \mathrm{E}-03$ & 0.0045 & -0.111 & 1178 & 2780 \\
\hline Left parahippocampal gyrus & -0.035 & 0.011 & -3.08 & $2.08 \mathrm{E}-03$ & 0.0060 & -0.106 & 1190 & 2850 \\
\hline Right transverse temporal gyrus & -0.025 & 0.008 & -3.05 & $2.30 \mathrm{E}-03$ & 0.0066 & -0.105 & 1190 & 2849 \\
\hline Left rostral middle frontal gyrus & -0.014 & 0.005 & -2.8 & $5.10 \mathrm{E}-03$ & 0.0140 & -0.097 & 1197 & 2848 \\
\hline Left rostral anterior cingulate cortex & -0.023 & 0.009 & -2.74 & $6.20 \mathrm{E}-03$ & 0.0165 & -0.095 & 1189 & 2835 \\
\hline Left medial orbitofrontal cortex & -0.015 & 0.006 & -2.51 & $1.22 \mathrm{E}-02$ & 0.0309 & -0.087 & 1182 & 2818 \\
\hline Left frontal pole & -0.028 & 0.011 & -2.49 & $1.28 \mathrm{E}-2$ & 0.0313 & -0.086 & 1199 & 2863 \\
\hline Right pars orbitalis & -0.020 & 0.008 & -2.5 & $1.26 \mathrm{E}-02$ & 0.0313 & -0.086 & 1198 & 2848 \\
\hline Left superior parietal cortex & -0.010 & 0.004 & -2.44 & $1.50 \mathrm{E}-02$ & 0.0350 & -0.084 & 1187 & 2831 \\
\hline Left pars orbitalis & -0.019 & 0.008 & -2.31 & $2.11 \mathrm{E}-02$ & 0.0473 & -0.080 & 1194 & 2854 \\
\hline \multicolumn{9}{|l|}{ Cortical surface area } \\
\hline Left isthmus cingulate cortex & 25.900 & 5.492 & 4.72 & $2.50 \mathrm{E}-06$ & $<0.0001$ & 0.167 & 1134 & 2700 \\
\hline Right isthmus cingulate cortex & 21.160 & 5.097 & 4.15 & $3.37 \mathrm{E}-05$ & 0.0002 & 0.147 & 1137 & 2706 \\
\hline Left transverse temporal gyrus & 10.183 & 2.603 & 3.91 & $9.32 \mathrm{E}-05$ & 0.0004 & 0.138 & 1141 & 2708 \\
\hline Right rostral middle frontal gyrus & -71.936 & 22.908 & -3.14 & $1.70 \mathrm{E}-03$ & 0.0050 & -0.111 & 1135 & 2698 \\
\hline Right paracentral lobule & 21.589 & 7.403 & 2.92 & $3.57 \mathrm{E}-03$ & 0.0100 & 0.104 & 1117 & 2688 \\
\hline Left inferior temporal gyrus & -40.910 & 15.209 & -2.69 & $7.18 \mathrm{E}-03$ & 0.0188 & -0.096 & 1099 & 2673 \\
\hline Right inferior temporal gyrus & -35.140 & 14.136 & -2.49 & $1.30 \mathrm{E}-02$ & 0.0313 & -0.089 & 1115 & 2684 \\
\hline Left paracentral lobule & 16.023 & 6.589 & 2.43 & $1.51 \mathrm{E}-02$ & 0.0350 & 0.087 & 1099 & 2658 \\
\hline Left lingual gyrus & -32.434 & 13.793 & -2.35 & $1.88 \mathrm{E}-02$ & 0.0428 & -0.083 & 1128 & 2692 \\
\hline \multicolumn{9}{|l|}{ Subcortical volume } \\
\hline Right amygdala & 41.656 & 6.984 & 5.96 & $2.68 \mathrm{E}-09$ & $<0.0001$ & 0.211 & 1129 & 2702 \\
\hline Left thalamus & 108.695 & 26.117 & 4.16 & $3.23 \mathrm{E}-05$ & 0.0002 & 0.147 & 1138 & 2691 \\
\hline Right thalamus & 80.814 & 22.216 & 3.64 & $2.80 \mathrm{E}-04$ & 0.0010 & 0.129 & 1134 & 2680 \\
\hline Left amygdala & 22.444 & 6.474 & 3.47 & $5.30 \mathrm{E}-04$ & 0.0018 & 0.123 & 1127 & 2694 \\
\hline Left nucleus accumbens & 11.724 & 3.541 & 3.31 & $9.40 \mathrm{E}-04$ & 0.0030 & 0.118 & 1110 & 2660 \\
\hline Right hippocampus & 33.557 & 13.810 & 2.43 & $1.52 \mathrm{E}-02$ & 0.0350 & 0.086 & 1136 & 2709 \\
\hline
\end{tabular}

Results are displayed for global measures, cortical thickness, and surface area as well as for subcortical volumes and sorted by $p$ value within each domain. All results are adjusted for age, sex, MDD diagnosis, and site. Regional surface and subcortical results are adjusted for total intracranial volume.

Estimate regression estimate, StdError standard error, $T$ t-value, $p$ uncorrected $p$ value, FDR adjusted $p$ FDR adjusted $p$ value, $N$ Obese number of obese subjects included in analysis, $N N W$ number of normal weight subjects included in analysis.

To rule out bias due to antidepressant medication intake in the MDD group, analyses were repeated by including current intake of antidepressant medication as additional nuisance regressor. Regional specificity of cortical thickness findings was assessed by conducting additional analyses accounting for mean cortical thickness. Highly similar results were observed in analyses controlling for the presence of antidepressant medication and in analyses adjusted for mean cortical thickness (Supplementary Tables 7, 8). Consistent results were observed in confirmatory analyses testing quadratic effects of BMI, in analyses accounting for quadratic effects of age and in analyses assessing the effect of weight group by including normal weight, overweight and obesity as categorial predictor (Supplementary Results and Supplementary Tables 9-11, Supplementary Fig. 5). Subsample analyses adjusting for head movement confirmed the overall pattern of results although obesityrelated brain structural abnormalities were attenuated in these analyses (Supplementary Results and Supplementary Table 12, Supplementary Fig. 5).

Similar regional effect sizes for the association between obesity and brain structural abnormalities in the left and right hemisphere could be observed in the present study (Supplementary Results), while descriptively larger effects were observed for the association between obesity and lower cortical thickness in the left compared with right cortical hemisphere. 

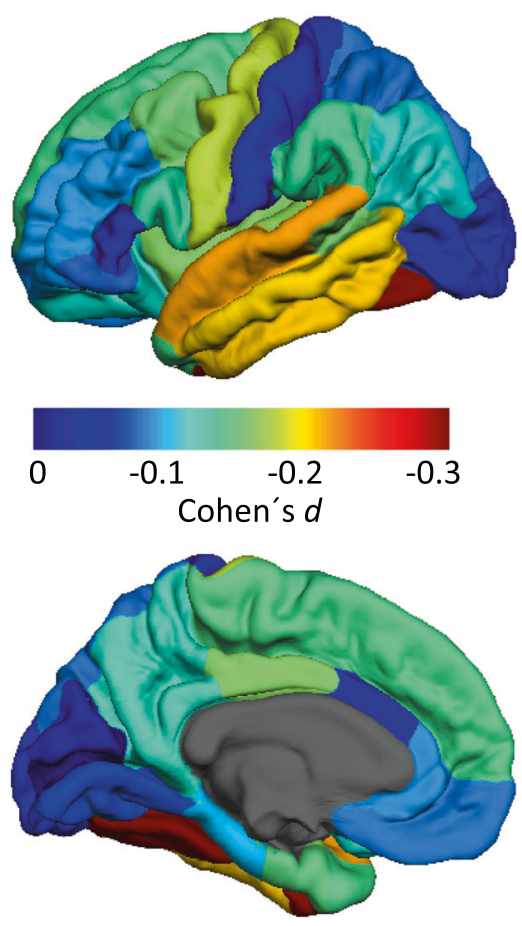

Fig. 1 Figure displaying effect sizes for the association between obesity and cortical thickness on left hemispheral thickness. Colorbar displays effect size estimates (Cohen's $d$ ) for differences in

\section{Comparison of obesity-related brain structural abnormalities with previous findings in neuropsychiatric disorders}

Correlational analyses of effect size estimates for thickness of each cortical region of interest indicated similarities in the relative distribution or pattern of cortical thickness reductions across cortical regions between obesity and MDD $(r=0.452)$ and obesity and bipolar disorder $(r=$ 0.513 ) (Fig. 2). An additional sensitivity analysis revealed that by contrast to the observed similarities between cortical thickness in obesity and affective disorders, effect sizes for obesity and previously published effect sizes for autism spectrum disorder (ASD) [35] did not show a similar degree of overlap ( $r=0.149)$ (Supplementary Results). Further analyses of the absolute extent of effect sizes for cortical thickness indicated overall larger effect sizes in obesity compared with MDD and ASD but lower effect sizes compared with BD (Supplementary Results, Supplementary Fig. 6).

\section{Multivariate pattern recognition analyses}

Multivariate pattern classification analyses further confirmed the relationship between obesity and brain structure by yielding highly significant single-subject differentiation cortical thickness between obese versus normal weight subjects; Bar diagram depicts effect sizes for all cortical regions sorted by lobe.

between obese (BMI $>30, n=1223)$ and normal weight subjects (BMI 18.5-25, $n=2917$ ) with a balanced accuracy rate of $68.7 \%(\mathrm{BAC}=0.687, \mathrm{StD}=0.019, p<0.001$; sensitivity $=0.695 ; \quad$ specificity $=0.678 ; \quad$ F1score $=0.565$; ROC-AUC $=0.687$ ).

To rule out bias due to differing age, sex, and MDD diagnosis distributions in obese versus normal weight subjects, pattern recognition analyses were repeated in samples of obese and normal weight subjects that were balanced for age, sex, and MDD diagnosis using the pairmatch function in $\mathrm{R}$ $\left(n_{\text {obese }}=1223 ; n_{\text {normal weight }}=1223\right)$. Similar results were observed when analyses were performed in samples of obese and normal weight subjects that were balanced for age, sex, and MDD diagnosis $\left(n_{\text {obese }}=1223 ; n_{\text {normal }}\right.$ weight $=1223$; $\mathrm{BAC}=0.641, \mathrm{StD}=0.014, p<0.001 ;$ sensitivity $=0.666$; specificity $=0.617$; F1score $=0.650 ;$ ROC-AUC $=0.641$ ).

In addition, to demonstrate replicability across differing cohorts and scanning sites, we performed pattern recognition analyses by employing leave-one-site-out crossvalidation. For this analysis step, only sites with a minimum of 50 subjects per group were included, to avoid bias due to lenient test sample sizes $\left(n_{\text {obese }}=960 ; n_{\text {normal weight }}=\right.$ 1616 ; $k=5$ sites). Analyses employing leave-one-site-outcross-validation including all sites with a minimum $n>50$ in each group yielded a lower but still highly significant accuracy rate $\left(n_{\text {obese }}=960 ; n_{\text {normal weight }}=1616, k=5\right.$ sites; 

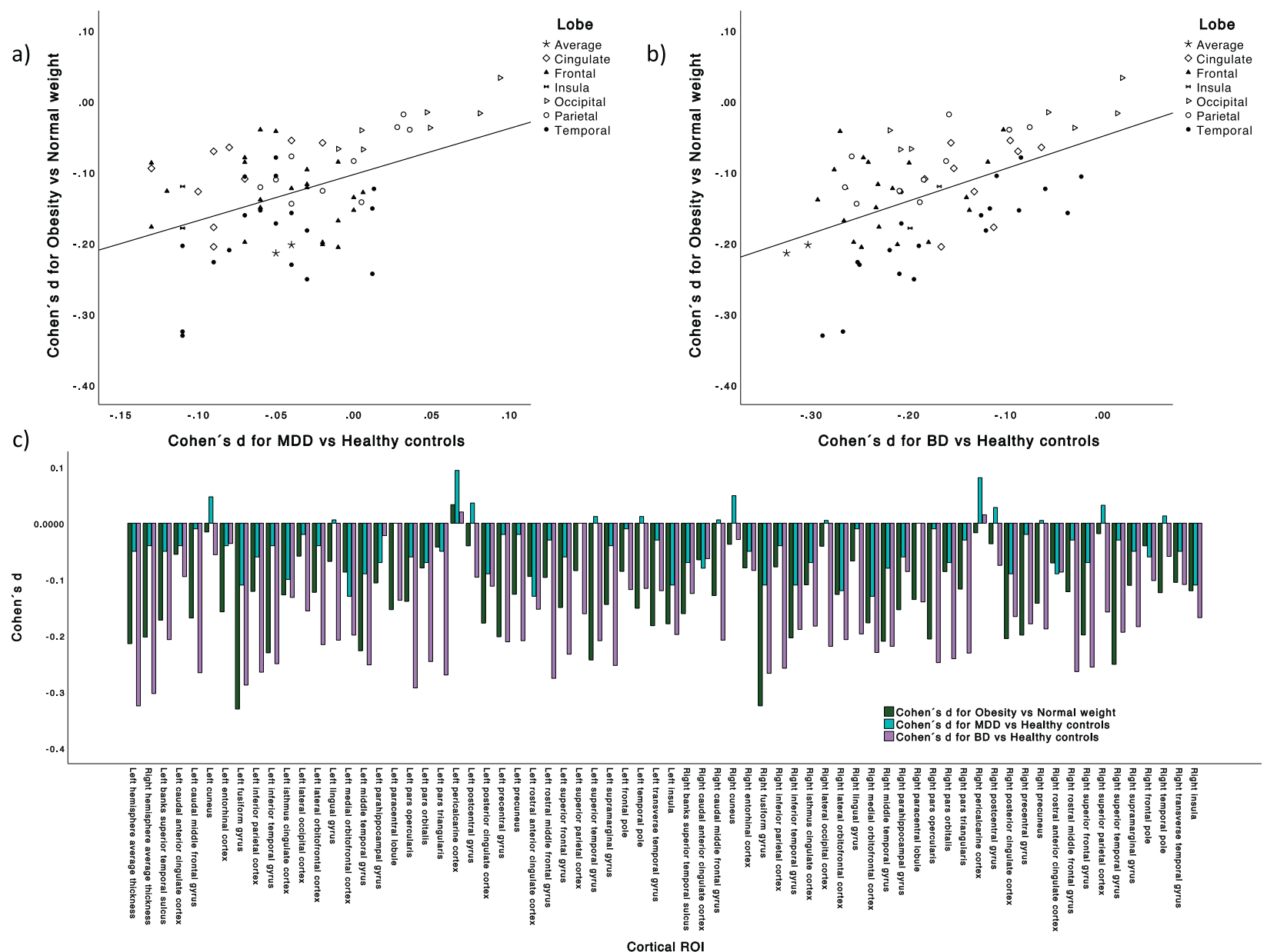

Fig. 2 Effect size estimates (Cohen's $d$ ) for differences in cortical thickness between obese versus normal weight subjects in direct comparison with previously published effect size estimates for cortical thickness results in major depression (MDD) and bipolar disorder (BD). a Plot depicting the positive correlation between effect

$\mathrm{BAC}=0.595, \mathrm{StD}=0.018, p<0.001 ;$ sensitivity $=0.714$; specificity $=0.476$; F1score $=0.523$; ROC-AUC $=0.595$ ).

Supplementary analyses confirmed the predictive relevance of brain regions associated with obesity in the univariate analyses but also revealed that optimal classifier performance was obtained in analyses including the maximum of available brain structural features (see Supplementary Results).

\section{Moderating role of MDD diagnosis, age, and sex}

To investigate if associations between BMI and brain structure would significantly differ between MDD and HC participants, interaction effects of BMI $\times$ MDD diagnosis were assessed based on linear models in analogy to Model $\mathrm{B}$ thus comparing slopes of $\mathrm{BMI} \times \mathrm{MRI}$ measure between MDD and HC subjects. No FDR-corrected significant interaction effect of BMI and MDD diagnosis was detected size estimates for thickness results in all cortical regions mapped to the respective lobe between obesity and MDD $(r=0.452)$ and $\mathbf{b}$ between obesity and $\mathrm{BD}(r=0.513)$. c Bar diagram displaying effect size estimates for cortical thickness results separately for all cortical regions.

(Supplementary Table 13). Similarly, analyses stratified by diagnostic group confirmed our main result by yielding significant associations between obesity and lower temporofrontal cortical thickness in both MDD and HC subjects with descriptively larger effect sizes for obesity in MDD compared with HC subjects (Supplementary Tables 14, 15, Supplementary Fig. 7).

Similarly, a moderating role of sex was investigated by assessing BMI $\times$ sex interaction effects. We observed FDRcorrected significant interaction effects of sex and BMI on cortical thickness, subcortical volumes, and surface area. The most consistent finding was a significantly enhanced BMI-related cortical thinning in male compared with female subjects (Supplementary Table 16).

To investigate a potential moderating role of age on brain structural alterations observed in obesity, linear models building on Model A were fitted by also including the obesity $\times$ age interaction term. FDR-corrected significant 
interaction effects of obesity and age were observed on cortical thickness of the left rostral middle frontal gyrus, the left lateral orbitofrontal gyrus, the left pars orbitalis, and triangularis of the inferior frontal gyrus driven by significantly enhanced age-related thickness decrease in obese compared with normal weight subjects. Further significant obesity $\times$ age interaction effects were observed for right hippocampal and left thalamic volume as well as for surface area of the right precuneus (Supplementary Table 17). Moreover, to investigate if brain structural associations with BMI could be detected in adolescents, regression analyses were repeated in the subgroup of participants with an age $<21(n=520)$. Due to the limited prevalence of obesity in the adolescent subgroup $(n=51)$, only models including BMI as continuous predictor were conducted in the adolescent subgroup. Additional subgroup analyses of associations between BMI and brain structure in adolescent participants exclusively revealed an FDR-corrected significant positive association between BMI and volume of the right amygdala $(B=$ $7.34, \mathrm{StdE}=1.72, t=4.26, p=0.00002, p_{(\mathrm{FDR})}=0.0038$, $n=503$ ) (Supplementary Table 18), while no further association reached FDR-corrected significance in this subsample.

\section{Polygenic risk for obesity and brain structure}

All calculated PRS scores significantly predicted BMI with proportions of explained variance $\left(\mathrm{R}^{2}\right)$ ranging from 1.2 to $1.8 \%(n=3907$, all $p<0.00001$; Supplementary Tables 19 , 20). To assess the influence of polygenic risk for obesity on brain structure, linear models were fitted (a) by including the PRS based on information from all available SNPs as predictor ( $p$ value threshold $=1.0)$ and $(\mathrm{b})$ by employing the polygenic score that explained most variance in BMI as predictor ( $p$ value threshold $=0.2$ )

We observed an FDR-corrected significant negative association between $\operatorname{PRS}_{(\mathrm{p} 1.0)}$ and cortical surface area of the left lateral occipital cortex $(B=-45.92, \mathrm{StdE}=12.56$, $\left.t=-3.66, p=0.00026, p_{(\mathrm{FDR})}=0.041, n=3526\right) \quad$ (Supplementary Table 21). Analyses including the $\mathrm{PRS}_{(\mathrm{p} 0.2)}$ as predictor yielded a highly similar pattern of results with the most pronounced association between polygenic risk and surface area of the left lateral occipital surface area, which, however, did not reach FDR-corrected significance $(B=$ $-40.84, \mathrm{StdE}=11.52, \quad t=-3.55, p=0.0004, \quad p_{(\mathrm{FDR})}=$ $0.062, n=3526$ ) (Supplementary Table 22). In addition, mediation analyses were performed to test if the association between polygenic risk and BMI was mediated by left lateral occipital surface area and other brain structures reported previously [24]. While we did not observe a significant mediation effect for left lateral occipital surface area, a significant mediation effect of polygenic risk for obesity on
BMI through left lateral orbitofrontal thickness could be detected (see Supplementary Results).

\section{Discussion}

In the present multisite study, we found that obesity significantly associated with cortical and subcortical brain structural abnormalities independent of MDD diagnosis in both univariate and multivariate analyses. We further demonstrate that the regional distribution and effect size of the observed lower cortical thickness in obesity shows considerable similarities with corresponding patterns of cortical thickness alterations that have been described in mental disorders. Similarly, the presence of differential age dependent effects on brain structural measures in obesityas well as the observed influence of polygenic risk for obesity on brain structure-offers novel insights of relevance for future experimental research on the etiology of obesity-related brain structural impairment.

The applied multisite design combined with a comprehensive neuroimaging approach allowed to differentiate between obesity-related abnormalities in cortical thickness, surface, and subcortical volume with unprecedented statistical power and detail. Our findings clarify that lower fronto-temporal cortical thickness constitutes the most pronounced obesity-related brain structural abnormality across the brain. This finding is supported by prior reports on temporal and frontal cortical gray matter decrease in obesity $[4,9,10,20,24,36]$.

Interestingly, while all significant associations between BMI and cortical thickness were negative, differing directions of associations occurred with regard to surface area alterations. This observation appears to match previously reported differential regionally specific positive and negative associations between cortical thickness and surface area $[29,37]$. A previously discussed explanation for the inverse relationship between cortical surface and thickness measures refers to a potential stretching of the cortical surface area along the tangential axis due to intracortical myelination [37, 38]. Our finding of larger subcortical volumes in obesity with strongest effects of greater amygdala, thalamic, nucleus accumbens, and hippocampal volume finds support in prior studies of obese subjects that applied a similar volumetric imaging approach reporting larger amygdala, thalamus, and hippocampal volumes [39, 40]. In contrast, previous voxel-based morphometry studies reported negative associations between BMI and gray matter of subcortical structures $[10,41]$. The disparity between volumetric and voxel-based findings has been directly investigated in a recent report by Perlaki et al. suggesting that BMI associates with higher amygdala and nucleus accumbens volumes derived from FreeSurfer segmentations 
but with lower VBM based GM density in identical structures highlighting the relevance to distinguish GM density from volume [13].

Importantly, we found that cortical thickness reductions in obesity are of similar effect size to the previously observed thickness reductions in several neuropsychiatric disorders. More specifically, peak effect sizes for lower cortical thickness in obesity (max. Cohen's $d$ (left fusiform gyrus $)=-0.331$ ) exceeded previously reported peak effect sizes for cortical thinning in MDD patients (max. Cohen's $d$ (left medial orbitofrontal cortex $)=-0.134$ ) [19], adult OCD patients (max Cohen's $d$ (right inferior parietal cortex $)=-0.140$ ) [42], findings in specific substance dependence (max Cohen's $d$ (right fusiform gyrus) $=-0.094$ ) [43] and were comparable with peak effect sizes in bipolar disorder (max Cohen's $d$ (left pars opercularis) $=-0.293$ ) [34]. Results of our pattern classification analyses further support the notion of a robust association between obesity and brain structure by yielding sMRI-based single-subject classification accuracies of up to $68.7 \%$ in pooled multisite cross-validation. Of note, this level of accuracy is comparable with pattern classification results reported for the detection of bipolar patients versus healthy controls using similar methods $(65.2 \%$ accuracy for support vector classifiers, trained on FreeSurfer segmentations using multisite pooled cross-validation) [44]. Similar to previous reports of accurate individual brain age prediction based on neuroanatomical data [45, 46], our findings highlight the importance to consider multivariate morphometric patterns related to phenotypes such as age and body weight in future pattern classification studies. Importantly, the presence of a multivariate pattern differentiating obese from normal weight subjects could similarly be demonstrated in analyses controlling for age, sex and MDD diagnosis and by transfer of the classifier across cohorts using leave-one-site-out-crossvalidation in the present work which underlines the robustness and the replicability of obesity-related brain structural abnormalities across sites. In addition, the relative distribution of obesity-related thickness reductions across all brain regions with most pronounced effects on temporofrontal cortical regions revealed considerable similarities with patterns of thickness reductions in major depression [19] and bipolar disorder [34] while the absolute extent of effect sizes across all regions in obesity was larger compared with MDD but lower compared with BD. In sum, these findings offer novel insights into shared brain structural abnormalities in obesity and affective disorders. In light of the known bidirectional association between obesity and affective disorders such as MDD [18], future studies should investigate the potential clinical relevance of the shared morphometric signature observed here.

Of note, no significant interaction of BMI and MDD diagnosis on brain structure was observed in the present work and similar obesity-related brain structural abnormalities emerged in separate analyses in the HD and MDD subsamples. We thus conclude that associations between brain structure and BMI are not significantly altered by the presence of depression. This is well in line with previous findings reporting similar associations between BMI and gray matter reductions in MDD patients and healthy subjects alike and no evidence for interaction effects of body weight and depression on brain structure [9, 47].

Furthermore, we observed that cortical thickness effects of obesity were significantly moderated by age. This interaction was driven by enhanced reductions of obesity-related cortical thickness with increasing age. Complementary to this notion, the most pronounced and significant associations between brain structure and BMI in adolescents were not observed in cortical regions but rather in the amygdala. Yet, it is important to acknowledge that BMI was associated with lower cortical thickness in adolescent participants but might have failed to reach significance due to limited sample size in this analysis (see Supplementary Results for power analysis). Regarding a potential explanation for early detectable amygdala volume increase in obesity, it appears important to consider the relevance of the amygdala in increased cue triggered learning [48] and Pavlovian conditioning to hedonic food that represents a key mechanism in future weight gain [49]. Importantly, the apparent discrepancy in obesity between early detectable subcortical volume increase on the one hand, and lower thickness with increasing age on the other, raises questions regarding potentially differing pathways behind the development of brain structural alterations in obesity that should be addressed by future experimental research.

The aforementioned notion of differing pathways underlying brain structural abnormalities in obesity appears to be further supplemented by the imaging genetic findings of the present study. The regionally pronounced effect of polygenic risk for obesity on lateral occipital surface area was unexpected. Prior studies have implicated the lateral occipital cortex in obesity [14, 50, 51], yet BMI was negatively correlated with occipital surface area but failed to reach significance in the present study $(p(\mathrm{FDR})=0.089)$. Similarly, since no significant mediation effect of lateral occipital surface area was observed in the association between polygenic risk and BMI, the functional relevance of this finding remains uncertain. In contrast, it appears important to note that in the present study left lateral orbitofrontal thickness mediated the association between polygenic risk and BMI which appears to replicate similar findings in a previous VBM study [24]. The notion that the influence of genetic risk for obesity on body weight might be mediated through changes in brain physiology is further supported by reports on high expression of obesity-related genes in the central-nervous system [23, 52]. Previous 
reports on associations between food addiction and OFC thickness [51] appear to further corroborate a model in which prefrontal brain regions might influence eating behavior and subsequent weight gain. However, results from these analyses have to be interpreted with great caution and do not allow for causal interpretations due to the cross-sectional design of the present study. Future studies are needed to directly test this hypothesis in experimental, longitudinal designs before form conclusions can be drawn.

Furthermore, it appears important to note that a large proportion of variance in obesity-related brain structural abnormalities could not be explained by genetic influence in the present study. It thus appears crucial to consider that increased body weight itself could contribute to brain structural abnormalities through mechanisms such as obesityrelated low-grade inflammation, kynurenine pathway activation, or neuroendocrine dysregulation [17, 53-55]. Another previously hypothesized link between obesity and brain structural abnormalities implies brain energy consumption during childhood and subsequent development of obesity [56], and hence points to educational interventions during childhood as a preventive measure against obesity.

Finally, the rather unexpected finding of a moderating role of sex on BMI-related cortical thickness decrease should be acknowledged. In the present study, male subjects exhibited significantly lower BMI-related cortical thickness compared with female participants. The potential relevance of this finding is highlighted by a previous PET study reporting significantly lower metabolic brain age in female compared with male subjects [57] and should be targeted by future research.

The presented analysis has strengths and limitations. Major strengths of the present work are the large sample size including healthy participants and depressive patients and the inclusion of imaging and genetic data. In addition, the combination of univariate group-level and multivariate machine learning techniques further highlighted the relevance of the observed associations on single-subject level. The most severe limitation of our study is the crosssectional design that prevents us from drawing causal conclusions. Our interpretations with regard to the onset and mechanisms behind brain structural abnormalities in obesity need clarification from longitudinal research before firm conclusions can be drawn. It furthermore appears important to note that BMI was not accounted for in previous studies on psychiatric disorders from the ENIGMA consortium. Considering the known association between affective disorders and obesity, the observed similarities between obesity and affective disorders observed here might thus partially be explained by higher BMI in the patient samples of such studies. Moreover, we acknowledge that our study sample is not independent from patient and control samples of previous ENIGMA studies and therefore overlap in participants might contribute to the similarities in brain structural findings between obesity and affective disorders.

To conclude, the present findings demonstrate similar associations between obesity and brain structural abnormalities in healthy participants and depressive patients. Cortical thickness reductions in the temporal and frontal cortex were identified as the most consistent and pronounced structural neuroimaging findings in adult obesity in the present study. Future voxel-wise neuroimaging studies capable of providing higher resolution should aim to further delineate the precise regional distribution of obesity-related gray matter decrease.

Results of the present study suggest that the distribution and extent of obesity-related brain structural abnormalities is comparable with findings in neuropsychiatric disorders. This notion critically underlines the similarities in patterns of impaired brain structural integrity between obesity and common neuropsychiatric disorders and points to the relevance of altered brain physiology in obesity that still appears to be drastically underestimated in current research. While neuropsychiatric disorders such as major depression are widely considered to be disorders of the brain, obesity is primarily considered as a cardiovascular risk factor in research and clinical practice. As the brain structural correlates of obesity exceed those of common neuropsychiatric disorders such as MDD_-in terms of affected regions and effect size per region-the findings presented here should urge clinicians and scientists to devote increased attention to neurobiological characteristics of obesity. The association of obesity with altered brain structural integrity in the present study indicates the need for a paradigm shift in obesity prevention and research.

Acknowledgements The ENIGMA-Major Depressive Disorder working group gratefully acknowledges support from the NIH Big Data to Knowledge (BD2K) award (U54 EB020403 to PT). LS is supported by a NHMRC Career Development Fellowship (1140764). PT is supported by grant R01MH116147. NJ is supported by grant R01MH117601. Muenster Cohort: The Muenster Neuroimaging Cohort was supported by grants of the German Research Foundation (DFG, grant FOR2107 DA1151/5-1 and DA1151/5-2 to UD; SFBTRR58, Projects C09 and Z02 to UD; ) and the Interdisciplinary Center for Clinical Research (IZKF) of the medical faculty of Münster (grant Dan3/012/17 to UD and SEED 11/18 to NO) and the Deanery of the Medical Faculty of the University of Münster. These funders had no role in designing the study; collection, management, analysis, and interpretation of data; writing of the report; nor the decision to submit the report for publication. FOR2107: The FOR2107 consortium is supported by the German Research Foundation (Deutsche Forschungsgemeinschaft, DFG, Grant nos. KI 588/14-1, KI 588/14-2, KR 3822/7-1, KR 3822/7-2, NE 2254/1-2, DA1151/5-1, DA1151/ 5-2, SCHW 559/14-1, 545/7-2, RI 908/11-2, WI 3439/3-2, NO 246/ 10-2, DE 1614/3-2, HA 7070/2-2, JA 1890/7-1, JA 1890/7-2, MU 1315/8-2, RE 737/20-2, KI 588/17-1). TH was supported by the German Research Foundation (DFG grants HA7070/2-2, HA7070/3, HA7070/4) and the Interdisciplinary Center for Clinical Research (IZKF) of the medical faculty of Münster (MzH3/020/20). BiDirect: The BiDirect-Study is funded by the German Federal Ministry 
of Education and Research (grants 01ER0816, 01ER1506 and 01ER1205). NESDA: Funding was obtained from the Netherlands Organization for Scientific Research (Geestkracht program grant 10-000-1002); the Center for Medical Systems Biology (CSMB, NWO Genomics), Biobanking and Biomolecular Resources Research Infrastructure (BBMRI-NL), VU University's Institutes for Health and Care Research (EMGO+) and Neuroscience Campus Amsterdam, University Medical Center Groningen, Leiden University Medical Center, National Institutes of Health (NIH, R01D0042157-01A, MH081802, Grand Opportunity grants 1RC2 MH089951 and 1RC2 MH089995). Part of the genotyping and analyses were funded by the Genetic Association Information Network (GAIN) of the Foundation for the National Institutes of Health.Computing was supported by BiG Grid, the Dutch e-Science Grid, which is financially supported by NWO. LS is supported by a NHMRC Career Development Fellowship (1140764) and grant R01MH117601. Bipolar Family Study: The Bipolar Family Study received funding from the European Union's Seventh Framework Programme for research under grant agreement $n^{\circ}$ 602450. This study is also supported by Wellcome Trust award 104036/Z/14/Z. CODE: The CODE cohort was collected from studies funded by Lundbeck and the German Research Foundation (WA 1539/ 4-1, SCHN 1204/3-1). MPIP: The MPIP Munich Morphometry Sample comprises patients included in Munich Antidepressant Response Signature study and the Recurrent Unipolar Depression (RUD) Case-Control study, and control subjects acquired at the Ludwig-Maximilians-University, Munich, Department of Psychiatry. We wish to acknowledge Rosa Schirmer, Elke Schreiter, Reinhold Borschke, and Ines Eidner for MR image acquisition and data preparation, and Benno Pütz, and Bertram Müller-Myhsok for distributed computing support and the MARS and RUD Study teams for clinical phenotyping. We thank Dorothee P. Auer for initiation of the RUD study. The MARS study is supported by a grant of the ExzellenzStiftung of the Max Planck Society. This work has also been funded by the Federal Ministry of Education and Research (BMBF) in the framework of the National Genome Research Network (NGFN), FKZ 01GS0481. SHIP: SHIP is part of the Community Medicine Research net of the University of Greifswald, Germany, which is funded by the Federal Ministry of Education and Research (grants no. 01ZZ9603, 01ZZ0103, and 01ZZ0403), the Ministry of Cultural Affairs and the Social Ministry of the Federal State of Mecklenburg-West Pomerania. MRI scans in SHIP and SHIP-TREND have been supported by a joint grant from Siemens Healthineers, Erlangen, Germany and the Federal State of Mecklenburg-West Pomerania. This study was further supported by the EU-JPND Funding for BRIDGET (FKZ:01ED1615). Stanford: NIMH Grant R37-101495 to IG, and the National Science Foundation Integrative Graduate Education and Research Traineeship (NSF IGERT) Recipient Award 0801700 and National Science Foundation Graduate Research Fellowship Program (NSF GRFP) DGE-1147470 to MS. Melbourne: The study was funded by National Health and Medical Research Council of Australia (NHMRC) Project Grants 1064643 (PI BH) and 1024570 (PI CD). Houston: Supported in part by NIMH grant R01 085667, The Dunn Foundation, and the Pat Rutherford, Jr. Endowed Chair in Psychiatry to JCS. Imaging genetics Dublin and Clinical Depression Dublin: The study was supported by a Science Foundation Ireland (SFI) Stokes Professorship Grant to Thomas Frodl. Novosibirsk: Russian Science Foundation grant \#16-15-00128 to Lyubomir Aftanas. Sydney: This study was supported by the following National Health \& Medical Research Council funding sources: Program Grant (No. 566529), Centres of Clinical Research Excellence Grant (No. 264611), Australia Fellowship (No. 464914) and Clinical Research Fellowship (No. 402864). Calgary: The study was supported by Branch Out Neurological Foundation, Alberta Children's Hospital Foundation. Magdeburg: The study was funded through the SFB779. Minnesota: The study was supported by K23MH090421, P41RR008079, National Alliance for Research on Schizophrenia and Affective Disorders, University of Minnesota
Graduate School, Minnesota Medical Foundation, Deborah E. Powell Center for Women's Health Seed Grant. Singapore: The study was supported by grant NHG SIG/15012.

\section{Compliance with ethical standards}

Conflict of interest HW and MH have previously received funding from The Sackler Trust. AM has previously received funding from Eli Lilly, Pfizer and The Sackler Trust. HG has received travel grants and speakers honoraria from Fresenius Medical Care, Neuraxpharm, Servier and Janssen Cilag as well as research funding from Fresenius Medical Care. PT is MPI of a research related grant from Biogen, Inc for work unrelated to this manuscript. NJ is MPI of a research related grant from Biogen, Inc for work unrelated to this manuscript. PGS has previously received funding by the German Research Foundation (DFG, SA 1358/2-1) unrelated to this study. These affiliations have no relevance to the work covered in the manuscript. The remaining authors declare no conflict of interest.

Publisher's note Springer Nature remains neutral with regard to jurisdictional claims in published maps and institutional affiliations.

Open Access This article is licensed under a Creative Commons Attribution 4.0 International License, which permits use, sharing, adaptation, distribution and reproduction in any medium or format, as long as you give appropriate credit to the original author(s) and the source, provide a link to the Creative Commons license, and indicate if changes were made. The images or other third party material in this article are included in the article's Creative Commons license, unless indicated otherwise in a credit line to the material. If material is not included in the article's Creative Commons license and your intended use is not permitted by statutory regulation or exceeds the permitted use, you will need to obtain permission directly from the copyright holder. To view a copy of this license, visit http://creativecommons. org/licenses/by/4.0/.

\section{References}

1. Blüher M. Obesity: global epidemiology and pathogenesis. Nat Rev Endocrinol. 2019;15:288-98.

2. World Health Organization. Obesity and overweight. 2014. http://www.who.int/mediacentre/factsheets/fs311/en/.

3. Raji CA, Ho AJ, Parikshak NN, Becker JT, Lopez OL, Kuller LH, et al. Brain structure and obesity. Hum Brain Mapp. 2010;31: 353-64.

4. Bobb JF, Schwartz BS, Davatzikos C, Caffo B. Cross-sectional and longitudinal association of body mass index and brain volume. Hum Brain Mapp. 2014;35:75-88.

5. Repple J, Opel N, Meinert S, Redlich R, Hahn T, Winter NR, et al. Elevated body-mass index is associated with reduced white matter integrity in two large independent cohorts. Psychoneuroendocrinology. 2018;91:179-85.

6. Opel N, Redlich R, Grotegerd D, Dohm K, Haupenthal C, Heindel $\mathrm{W}$, et al. Enhanced neural responsiveness to reward associated with obesity in the absence of food-related stimuli. Hum Brain Mapp. 2015;36:2330-7.

7. Stice E, Yokum S, Burger KS, Epstein LH, Small DM. Youth at risk for obesity show greater activation of striatal and somatosensory regions to food. J Neurosci. 2011;31:4360-6.

8. Burger KS, Berner LA. A functional neuroimaging review of obesity, appetitive hormones and ingestive behavior. Physiol Behav. 2014. https://doi.org/10.1016/j.physbeh.2014.04.025.

9. Opel N, Redlich R, Grotegerd D, Dohm K, Heindel W, Kugel H, et al. Obesity and major depression: body-mass index (BMI) 
is associated with a severe course of disease and specific neurostructural alterations. Psychoneuroendocrinology. 2015;51: 219-26.

10. Janowitz D, Wittfeld K, Terock J, Freyberger HJ, Hegenscheid K, Völzke H, et al. Association between waist circumference and gray matter volume in 2344 individuals from two adult community-based samples. Neuroimage. 2015;122:149-57.

11. Hamer M, Batty GD. Association of body mass index and waistto-hip ratio with brain structure. Neurology. 2019. https://doi.org/ 10.1212/WNL.0000000000006879.

12. García-García I, Michaud A, Dadar M, Zeighami Y, Neseliler S, Collins DL, et al. Neuroanatomical differences in obesity: metaanalytic findings and their validation in an independent dataset. Int J Obes. 2018. https://doi.org/10.1038/s41366-018-0164-4.

13. Perlaki G, Molnar D, Smeets PAM, Ahrens W, Wolters M, Eiben $\mathrm{G}$, et al. Volumetric gray matter measures of amygdala and accumbens in childhood overweight/obesity. PLoS One. 2018;13: e0205331.

14. Medic N, Ziauddeen H, Ersche KD, Farooqi IS, Bullmore ET, Nathan PJ, et al. Increased body mass index is associated with specific regional alterations in brain structure. Int J Obes. 2016; 40:1177-82.

15. Reddan MC, Lindquist MA, Wager TD. Effect size estimation in neuroimaging. JAMA Psychiatry. 2017;74:207.

16. Simon GE, Von Korff M, Saunders K, Miglioretti DL, Crane PK, van Belle G, et al. Association between obesity and psychiatric disorders in the US adult population. Arch Gen Psychiatry. 2006; 63:824.

17. Milaneschi Y, Simmons WK, van Rossum EFC, Penninx BW. Depression and obesity: evidence of shared biological mechanisms. Mol Psychiatry. 2019;24:18-33.

18. Luppino F, de Wit LM, Bouvy PF, Stijnen T, Cuijpers P, Penninx $\mathrm{B}$, et al. Overweight, obesity, and depression. Arch Gen Psychiatry. 2010;67:220-9.

19. Schmaal L, Hibar DP, Sämann PG, Hall GB, Baune BT, Jahanshad N, et al. Cortical abnormalities in adults and adolescents with major depression based on brain scans from 20 cohorts worldwide in the ENIGMA Major Depressive Disorder Working Group. Mol Psychiatry. 2017;22:900-9.

20. Marqués-Iturria I, Pueyo R, Garolera M, Segura B, Junqué C, García-García I, et al. Frontal cortical thinning and subcortical volume reductions in early adulthood obesity. Psychiatry Res Neuroimaging. 2013;214:109-15.

21. Sharkey RJ, Karama S, Dagher A. Overweight is not associated with cortical thickness alterations in children. Front Neurosci. 2015;9:24.

22. Stunkard AJ, Harris JR, Pedersen NL, McClearn GE. The bodymass index of twins who have been reared apart. N. Engl J Med. 1990;322:1483-7.

23. Locke AE, Kahali B, Berndt SI, Justice AE, Pers TH, Day FR, et al. Genetic studies of body mass index yield new insights for obesity biology. Nature. 2015;518:197-206.

24. Opel N, Redlich R, Kaehler C, Grotegerd D, Dohm K, Heindel W, et al. Prefrontal gray matter volume mediates genetic risks for obesity. Mol Psychiatry. 2017;22:703-10.

25. Wolf EJ, Miller DR, Logue MW, Sumner J, Stoop TB, Leritz EC, et al. Contributions of polygenic risk for obesity to PTSD-related metabolic syndrome and cortical thickness. Brain Behav Immun. 2017;65:328-36.

26. Schmaal L, Veltman DJ, van Erp TGM, Sämann PG, Frodl T, Jahanshad N, et al. Subcortical brain alterations in major depressive disorder: findings from the ENIGMA Major Depressive Disorder working group. Mol Psychiatry. 2016;21:806-12.

27. Fischl B, Salat DH, Busa E, Albert M, Dieterich M, Haselgrove C, et al. Whole brain segmentation: automated labeling of neuroanatomical structures in the human brain. Neuron. 2002;33:341-55.
28. Desikan RS, Ségonne F, Fischl B, Quinn BT, Dickerson BC, Blacker D, et al. An automated labeling system for subdividing the human cerebral cortex on MRI scans into gyral based regions of interest. Neuroimage. 2006;31:968-80.

29. Storsve AB, Fjell AM, Tamnes CK, Westlye LT, Overbye K, Aasland HW, et al. Differential longitudinal changes in cortical thickness, surface area and volume across the adult life span: regions of accelerating and decelerating change. $J$ Neurosci. 2014;34:8488-98.

30. Winkler AM, Kochunov P, Blangero J, Almasy L, Zilles K, Fox PT, et al. Cortical thickness or grey matter volume? The importance of selecting the phenotype for imaging genetics studies. Neuroimage. 2010;53:1135-46.

31. Euesden J, Lewis CM, O'Reilly PF. PRSice: polygenic risk score software. Bioinformatics. 2015;31:1466-8.

32. Chang CC, Chow CC, Tellier LC, Vattikuti S, Purcell SM, Lee JJ. Second-generation PLINK: rising to the challenge of larger and richer datasets. Gigascience. 2015;4:7.

33. Rosenthal R, Rosnow RL. Essentials of Behavioral Research: Methods Data Anal. 2008.

34. Hibar DP, Westlye LT, Doan NT, Jahanshad N, Cheung JW, Ching CRK, et al. Cortical abnormalities in bipolar disorder: an MRI analysis of 6503 individuals from the ENIGMA Bipolar Disorder Working Group. Mol Psychiatry. 2018;23:932-42.

35. van Rooij D, Anagnostou E, Arango C, Auzias G, Behrmann M, Busatto GF, et al. Cortical and subcortical brain morphometry differences between patients with autism spectrum disorder and healthy individuals across the lifespan: results from the ENIGMA ASD Working Group. Am J Psychiatry. 2018;175:359-69.

36. Ronan L, Alexander-Bloch AF, Wagstyl K, Farooqi S, Brayne C, Tyler LK, et al. Obesity associated with increased brain age from midlife. Neurobiol Aging. 2016;47:63-70.

37. Hogstrom LJ, Westlye LT, Walhovd KB, Fjell AM. The structure of the cerebral cortex across adult life: age-related patterns of surface area, thickness, and gyrification. Cereb Cortex. 2013; 23:2521-30.

38. Seldon HL. Does brain white matter growth expand the cortex like a balloon? Hypothesis and consequences. Laterality Asymmetries Body Brain Cogn. 2005;10:81-95.

39. Bernardes G, IJzerman RG, Ten Kulve JS, Barkhof F, Diamant M, Veltman DJ, et al. Cortical and subcortical gray matter structural alterations in normoglycemic obese and type 2 diabetes patients: relationship with adiposity, glucose, and insulin. Metab Brain Dis. 2018;33:1211-22.

40. Widya RL, De Roos A, Trompet S, De Craen AJM, Westendorp RGJ, Smit JWA, et al. Increased amygdalar and hippocampal volumes in elderly obese individuals with or at risk of cardiovascular disease. Am J Clin Nutr. 2011;93:1190-5.

41. Kharabian Masouleh S, Arélin K, Horstmann A, Lampe L, Kipping JA, Luck T, et al. Higher body mass index in older adults is associated with lower gray matter volume: implications for memory performance. Neurobiol Aging. 2016;40:1-10.

42. Boedhoe PSW, Schmaal L, Abe Y, Alonso P, Ameis SH, Anticevic A, et al. Cortical abnormalities associated with pediatric and adult obsessive-compulsive disorder: findings from the ENIGMA Obsessive-Compulsive Disorder Working Group. Am J Psychiatry. 2018;175:453-62.

43. Mackey S, Allgaier N, Chaarani B, Spechler P, Orr C, Bunn J, et al. Mega-analysis of gray matter volume in substance dependence: general and substance-specific regional effects. Am J Psychiatry. 2019;176:119-28.

44. Nunes A, Schnack HG, Ching CRK, Agartz I, Akudjedu TN, Alda $M$ et al. Using structural MRI to identify bipolar disorders13 site machine learning study in 3020 individuals from the ENIGMA Bipolar Disorders Working Group. Mol Psychiatry. 2018. https://doi.org/10.1038/s41380-018-0228-9. [Epub ahead of print]. 
45. Cole JH, Poudel RPK, Tsagkrasoulis D, Caan MWA, Steves C, Spector TD, et al. Predicting brain age with deep learning from raw imaging data results in a reliable and heritable biomarker. Neuroimage. 2017;163:115-24.

46. Koutsouleris N, Davatzikos C, Borgwardt S, Gaser C, Bottlender $\mathrm{R}$, Frodl $\mathrm{T}$, et al. Accelerated brain aging in schizophrenia and beyond: a neuroanatomical marker of psychiatric disorders. Schizophr Bull. 2014;40:1140-53.

47. Cole JH, Boyle CP, Simmons A, Cohen-Woods S, Rivera M, McGuffin P, et al. Body mass index, but not FTO genotype or major depressive disorder, influences brain structure. Neuroscience. 2013; 252:109-17.

48. Rangel A. Regulation of dietary choice by the decision-making circuitry. Nat Neurosci. 2013;16:1717-24.

49. Meyer MD, Risbrough VB, Liang J, Boutelle KN. Pavlovian conditioning to hedonic food cues in overweight and lean individuals. Appetite. 2015;87:56-61.

50. Veit R, Kullmann S, Heni M, Machann J, Häring H-U, Fritsche A, et al. Reduced cortical thickness associated with visceral fat and BMI. NeuroImage Clin. 2014;6:307-11.

51. Beyer F, García-García I, Heinrich M, Schroeter ML, Sacher J, Luck T, et al. Neuroanatomical correlates of food addiction symptoms and body mass index in the general population. Hum Brain Mapp. 2019;40:2747-58.

52. Willer CJ, Speliotes EK, Loos RJF, Li S, Lindgren CM, Heid IM, et al. Six new loci associated with body mass index highlight a neuronal influence on body weight regulation. Nat Genet. 2009; 41:25-34.

53. Ho JE, Larson MG, Ghorbani A, Cheng S, Chen M-H, Keyes M, et al. Metabolomic profiles of body mass index in the framingham heart study reveal distinct cardiometabolic phenotypes. PLoS One. 2016;11:e0148361.

54. Favennec M, Hennart B, Caiazzo R, Leloire A, Yengo L, Verbanck $\mathrm{M}$, et al. The kynurenine pathway is activated in human obesity and shifted toward kynurenine monooxygenase activation. Obesity. 2015;23:2066-74.

55. Schwarcz R, Bruno JP, Muchowski PJ, Wu H-Q. Kynurenines in the mammalian brain: when physiology meets pathology. Nat Rev Neurosci. 2012;13:465-77.

56. Kuzawa $\mathrm{CW}$, Blair C. A hypothesis linking the energy demand of the brain to obesity risk. Proc Natl Acad Sci. 2019;116:13266-75.

57. Goyal MS, Blazey TM, Su Y, Couture LE, Durbin TJ, Bateman $\mathrm{RJ}$, et al. Persistent metabolic youth in the aging female brain. Proc Natl Acad Sci USA. 2019;116:3251-5.

\section{Affiliations}

Nils Opel ${ }^{1,2} \cdot$ Anbupalam Thalamuthu $^{3,4} \cdot$ Yuri Milaneschi $^{5} \cdot$ Dominik Grotegerd $^{1}$ - Claas Flint $\mathbb{D}^{1,6}$. Ramona Leenings $^{1}$. Janik Goltermann ${ }^{1} \cdot$ Maike Richter $^{1} \cdot$ Tim Hahn $^{1} \cdot$ Georg Woditsch $^{7} \cdot$ Klaus Berger $^{8}$. Marco Hermesdorf ${ }^{8}$. Andrew McIntosh $\mathbb{D}^{9} \cdot$ Heather C. Whalley $\mathbb{D}^{9} \cdot$ Mathew A. Harris $\mathbb{D}^{9} \cdot$ Frank P. MacMaster $^{10,11}$. Henrik Walter $\mathbb{D}^{12} \cdot$ Ilya M. Veer ${ }^{12} \cdot$ Thomas Frodl $^{13,14} \cdot$ Angela Carballedo $^{13} \cdot$ Axel Krug $^{15} \cdot$ Igor Nenadic $^{15}$. Tilo Kircher ${ }^{15}$ - Andre Aleman ${ }^{16} \cdot$ Nynke A. Groenewold ${ }^{16} \cdot$ Dan J. Stein $\unrhd^{17}$ - Jair C. Soares ${ }^{18}$. Giovana B. Zunta-Soares ${ }^{18} \cdot$ Benson Mwangi ${ }^{19} \cdot$ Mon-Ju Wu $\mathbb{D}^{19} \cdot$ Martin Walter $^{20} \cdot$ Meng Li $^{20} \cdot$ Ben J. Harrison ${ }^{21}$. Christopher G. Davey $\mathbb{1}^{22,23} \cdot$ Kathryn R. Cullen ${ }^{24}$ - Bonnie Klimes-Dougan ${ }^{25} \cdot$ Bryon A. Mueller $\mathbb{1}^{24}$.

Philipp G. Sämann ${ }^{26} \cdot$ Brenda Penninx $^{5} \cdot$ Laura Nawijn $\mathbb{1}^{5} \cdot$ Dick J. Veltman ${ }^{5}$ Lyubomir Aftanas ${ }^{27} \cdot$ Ivan V. Brak $\mathbb{1}^{27}$. Elena A. Filimonova ${ }^{27}$ - Evgeniy A. Osipov $\mathbb{D}^{28}$ - Liesbeth Reneman ${ }^{29} \cdot$ Anouk Schrantee $^{29} \cdot$ Hans J. Grabe $\mathbb{D}^{30,31}$. Sandra Van der Auwera ${ }^{30,31}$ - Katharina Wittfeld $\mathbb{D}^{30,31}$. Norbert Hosten ${ }^{32}$. Henry Völzke ${ }^{33,34} \cdot$ Kang Sim $\mathbb{B}^{35,36}$. Ian H. Gotlib ${ }^{37}$ - Matthew D. Sacchet ${ }^{38}$ - Jim Lagopoulos ${ }^{39}$. Sean N. Hatton ${ }^{40}{ }^{40}$ Ian Hickie ${ }^{40}$ - Elena Pozzi ${ }^{22,41}$. Paul M. Thompson ${ }^{42} \cdot$ Neda Jahanshad $\mathbb{1}^{42} \cdot$ Lianne Schmaal ${ }^{22,23} \cdot$ Bernhard T. Baune $^{1,43,44} \cdot$ Udo Dannlowski $^{1}$

1 Department of Psychiatry, University of Münster, Münster, Germany

2 Interdisciplinary Centre for Clinical Research (IZKF), University of Münster, Münster, Germany

3 Centre for Healthy Brain Ageing, School of Psychiatry, University of New South Wales, Sydney, NSW, Australia

4 Neuroscience Research Australia, Randwick, NSW, Australia

5 Department of Psychiatry, Amsterdam UMC/Vrije Universiteit, Amsterdam, Netherlands

6 Faculty of Mathematics and Computer Science, University of Münster, Münster, Germany

7 IT Department, University of Muenster, Münster, Germany

8 Institute of Epidemiology and Social Medicine, University of Münster, Münster, Germany

9 Division of Psychiatry, University of Edinburgh, Edinburgh, UK
10 Psychiatry and Paediatrics, University of Calgary, Calgary, AB, Canada

11 Addictions and Mental Health Strategic Clinical Network Calgary, Calgary, AB, Canada

12 Division of Mind and Brain Research, Department of Psychiatry and Psychotherapy CCM, Charité - Universitätsmedizin Berlin, corporate member of Freie Universität Berlin, HumboldtUniversität zu Berlin, and Berlin Institute of Health, Berlin, Germany

13 Department of Psychiatry, Trinity College Dublin, Dublin, Ireland

14 Department of Psychiatry and Psychotherapy, Otto von Guericke University Magdeburg, Magdeburg, Germany

15 Department of Psychiatry and Psychotherapy, University of Marburg, Marburg, Germany

16 Department of Biomedical Sciences of Cells and Systems, University of Groningen, University Medical Center Groningen, Groningen, The Netherlands 
17 SA MRC Unit on Risk \& Resilience, Department of Psychiatry and Neuroscience Institute, University of Cape Town, Cape Town, South Africa

18 UT Center of Excellence on Mood Disorders, Department of Psychiatry and Behavioral Sciences, University of Texas Health Science Center at Houston, Houston, TX, USA

19 Department of Psychiatry, University of Texas Health Science Center at Houston, Houston, TX, USA

Department of Psychiatry and Psychotherapy, Jena University Hospital, Jena, Germany

21 Melbourne Neuropsychiatry Centre, Department of Psychiatry, The University of Melbourne \& Melbourne Health, Parkville, VIC, Australia

22 Orygen, The National Centre of Excellence in Youth Mental Health, Parkville, VIC, Australia

23 Centre for Youth Mental Health, The University of Melbourne, Parkville, VIC, Australia

Department of Psychiatry and Behavioral Sciences, School of Medicine, University of Minnesota, Minneapolis, MN, USA

25

Department of Psychology, University of Minnesota, Minneapolis, MN, USA

26 Max Planck Institute of Psychiatry, Munich, Germany

27 FSSBI "Scientific Research Institute of Physiology \& Basic Medicine", Laboratory of Affective, Cognitive \& Translational Neuroscience, Novosibirsk, Russia

Novosibirsk State University, Laboratory of Experimental \& Translational Neuroscience, Novosibirsk, Russia

29 Department of Radiology and Nuclear Medicine, Academic Medical Center, University of Amsterdam,

Amsterdam, Netherlands
30 Department of Psychiatry and Psychotherapy, University Medicine Greifswald, Greifswald, Germany

31 German Center for Neurodegenerative Diseases (DZNE), Greifswald/Rostock, site Greifswald, Greifswald, Germany

32 Institute of Diagnostic Radiology and Neuroradiology, University Medicine Greifswald, Greifswald, Germany

33 Institute for Community Medicine, University Medicine Greifswald, Greifswald, Germany

34 German Center for Cardiovascular Research (DZHK), Partner Site Greifswald, Greifswald, Germany

35 West Region, Institute of Mental Health, Singapore, Singapore

36 Yoo Loo Lin School of Medicine, National University of Singapore, Singapore, Singapore

37 Department of Psychology, Stanford University, Stanford, CA, USA

38 Center for Depression, Anxiety, and Stress Research, McLean Hospital, Harvard Medical School, Belmont, MA, USA

39 Sunshine Coast Mind and Neuroscience Thompson Institute, University of the Sunshine Coast, Sippy Downs, QLD, Australia

40 Brain and Mind Centre, University of Sydney, Camperdown, NSW, Australia

41 Melbourne Neuropsychiatry Centre, Department of Psychiatry, The University of Melbourne, Parkville, VIC, Australia

42 Mark \& Mary Stevens Neuroimaging \& Informatics Institute, Keck School of Medicine, University of Southern California, Marina del Rey, CA, USA

43 Department of Psychiatry, Melbourne Medical School, The University of Melbourne, Melbourne, VIC, Australia

44 The Florey Institute of Neuroscience and Mental Health, The University of Melbourne, Parkville, VIC, Australia 